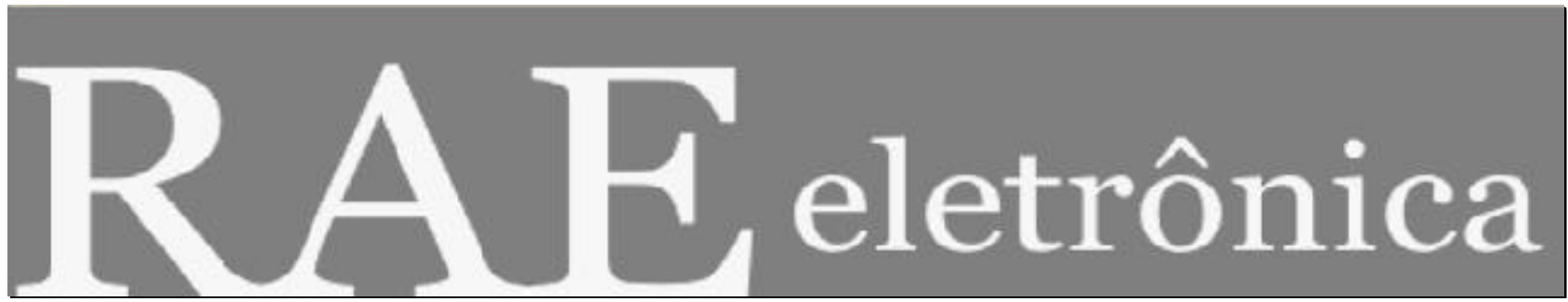

\title{
INCUBADORA DE INOVAÇÃO: QUE NOVA ESPÉCIE É ESSA? ${ }^{1}$
}

Por:

\section{Conceição Vedovello \\ Paulo N. Figueiredo}

RAE-eletrônica, v. 4, n. 1, Art. 10, jan./jul. 2005

http://www.rae.com.br/eletronica/index.cfm?FuseAction=Artigo \&ID=2363\&Secao=ARTIGOS\&Volu $\mathrm{me}=4 \&$ Numero $=1 \&$ Ano $=2005$

CCopyright, 2005, RAE-eletrônica. Todos os direitos, inclusive de tradução, são reservados. É permitido citar parte de artigos sem autorização prévia desde que seja identificada a fonte. A reprodução total de artigos é proibida. Os artigos só devem ser usados para uso pessoal e nãocomercial. Em caso de dúvidas, consulte a redação: raeredacao@fgvsp.br.

A RAE-eletrônica é a revista on-line da FGV-EAESP, totalmente aberta e criada com o objetivo de agilizar a veiculação de trabalhos inéditos. Lançada em janeiro de 2002, com perfil acadêmico, é dedicada a professores, pesquisadores e estudantes. Para mais informações consulte o site www.rae.com.br/eletronica.

\section{RAE-eletrônica}

\section{ISSN 1676-5648}

(C2005 Fundação Getulio Vargas - Escola de Administração de Empresas de São Paulo.

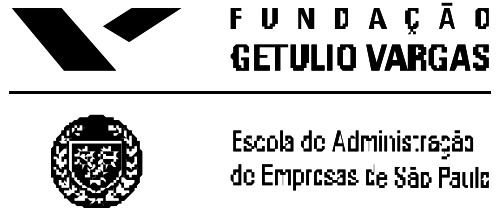




\title{
RESUMO
}

Este artigo enfoca a transferência de conhecimento da infra-estrutura tecnológica para a indústria, a partir da experiência de uma incubadora de inovação - modalidade rara de incubadora que nasce dentro de uma corporação e torna-se independente. Esta experiência é aqui examinada por meio do Genius Instituto de Tecnologia, nascido a partir da Gradiente Eletrônica S/A. Optando pela estratégia de estudo de caso individual, este artigo baseia-se em evidências empíricas qualitativas coletadas através de técnicas múltiplas (entrevistas formais, reuniões casuais, observação direta e consulta à documentação) durante trabalho de campo original e detalhado. Além de expandir o conhecimento sobre o papel da inovação na estratégia empresarial, este estudo contribui para adicionar novos elementos às tipologias sobre incubadoras - incubadora de inovação - onde cabe destacar o papel central dos recursos humanos altamente qualificados e do estabelecimento de parcerias como bases de sua sustentação.

\section{PALAVRAS CHAVE}

Transferência de conhecimento, infra-estrutura tecnológica, incubadora de inovação, estratégia de inovação.

\begin{abstract}
This article focuses on knowledge transfer from technological infrastructure to industry, from the perspective of an innovation incubator - a rare category of incubator that starts up inside of a corporation and becomes independent. This experience is examined through the Genius Institute of Technology, a spin-off from Gradiente Eletrônica S/A. Opting for a single case-study strategy, this article is based on empirical evidence, mainly qualitative, gathered from multiple sources (formal interviews, casual meetings, direct observation, and search into the company archival records) on the basis of detailed fieldwork. Besides expanding our knowledge on the role of innovation in business strategies, this study also contributes to adding new elements to existing typologies of technological incubators - innovation incubator - in which highly qualified human resources and the establishment of synergies are the bases for its sustainable development.
\end{abstract}

\section{KEYWORDS}

Knowledge transfer, technological infrastructure, innovation incubator, innovation strategies. 


\section{INTRODUÇÃO}

Nos anos recentes, a infra-estrutura tecnológica tem despertado crescente interesse em diversos segmentos políticos e sócio-econômicos, incluindo tomadores de decisão, agências de desenvolvimento, empreendedores, e a comunidade acadêmica e de pesquisa. $\mathrm{O}$ foco das políticas industrial e de inovação tem, gradualmente, mudado de um uso dominante e/ou exclusivo de instrumentos diretos de apoio para outras formas mais indiretas. Com o objetivo de melhorar o ambiente competitivo das empresas, esforços consideráveis têm sido canalizados para a construção e reforço da infra-estrutura tecnológica, em particular na implementação e desenvolvimento de incubadoras de empresas.

Incubadoras de empresas, como parte da infra-estrutura tecnológica, têm sido examinadas na literatura internacional, de forma mais sistemática, a partir dos anos 1970. O foco de análise dos estudos empíricos que emergiram naquela época era voltado, principalmente, para a observação das implicações de incubadoras na geração de empregos, na revitalização de áreas economicamente deprimidas e como um dos mecanismos de interação entre o setor acadêmico e o setor industrial (ver, por exemplo, Aydalot \& Keeble, 1988; Cohen \& Simmie, 1991; Currie, 1985; Dorfman, 1983; Lalkaka, 1997; Massey et al, 1992).

Com o aprofundamento do entendimento do processo de inovação tecnológica, a partir dos anos 1980, as expectativas em relação às incubadoras ampliam-se, buscando captar, além dos objetivos acima mencionados, elementos de caráter intangível. Como enfatizado no relatório do Banco Mundial (World Bank, 1999), as economias não são mais construídas exclusivamente pela acumulação física de capital e de recursos humanos, mas contam também com insumos mais intangíveis, como informação, conhecimento, aprendizado e adaptação.

À luz dessa perspectiva, novos estudos empíricos emergiram na literatura internacional. Tais estudos enfocam incubadoras de empresas como um dos vários mecanismos-chave da infra-estrutura tecnológica para a disseminação de atividades inovadoras no setor produtivo na chamada economia do conhecimento (ver, por exemplo, Lundvall et al., 2002; Vedovello \& Godinho, 2003; Zedwitz, 2003).

A questão das incubadoras de empresas passa a ser incorporada na agenda de política no Brasil a partir de meados da década de 1980 e, mais fortemente, a partir dos anos 1990. Entretanto, tanto na literatura internacional, como na nacional, observamse limitações quanto às abordagens apresentadas. Por exemplo, na literatura internacional, o European Innovation Monitoring System - EIMS (1996) observa incubadoras de empresas a partir das principais características tecnológicas das empresas que elas abrigam, ou seja, mais vocacionadas para a interação com universidades, ou mais relacionadas com o desenvolvimento regional. No contexto brasileiro, de maneira similar, a Associação Nacional de Entidades Promotoras de Empreendimentos de Tecnologias Avançadas (ANPROTEC) identifica incubadoras como tecnológicas, tradicionais, mistas ou outras (culturais, cooperativas, agroindustriais) (ANPROTEC, 2004). A despeito dos méritos dos estudos existentes, o seu foco de análise e as taxonomias apresentadas nessas abordagens não captam tipos de incubadora que divergem das características e classificações usuais.

Adicionalmente, no que diz respeito à implantação e operacionalização de incubadoras em economias emergentes, nota-se, ainda, um agravante: as incubadoras têm sido implementadas à luz de modelos 
adotados em países desenvolvidos, cuja infra-estrutura tecnológica já se encontra consolidada e a preocupação é com o seu aprimoramento contínuo. Porém, no contexto de economias emergentes, grande parte da infra-estrutura tecnológica encontra-se em processo de construção e consolidação. Nesse sentido, as incubadoras de empresas são, normalmente, implantadas objetivando prover as suas empresas incubadas de estrutura física, ou seja, aspectos tangíveis. Aspectos outros, como por exemplo, a natureza dos vínculos com os demais componentes da infra-estrutura tecnológica e a absorção sustentada de recursos humanos qualificados não têm sido, normalmente, tratados como uma questão estratégica.

Portanto, o foco deste estudo volta-se não para incubadoras de empresas no sentido como são convencionalmente examinadas, tal como mencionado acima. O foco aqui é uma modalidade de incubadora que nasce dentro de uma corporação, torna-se independente e se transforma em uma incubadora de inovação. Este tipo de incubadora dedica-se à geração de inovações que são oferecidas ao mercado, tendo como base de sua operacionalização o estabelecimento e fortalecimento de sinergias com a infra-estrutura tecnológica existente e empresas, combinado com a absorção e manutenção de recursos humanos altamente qualificados.

Mais especificamente, convém clarificar que, no sentido convencional do termo, as incubadoras de empresas abrigam negócios - inovadores ou não - que geram novos produtos ou processos ou equipamentos que serão produzidos e comercializados diretamente por eles. Isto, porém, não é o caso da modalidade de incubadora de inovação que examinamos aqui por meio da experiência do Genius Instituto de Tecnologia - doravante Genius - localizado em Manaus, Estado do Amazonas. O Genius, fruto de uma iniciativa da Gradiente Eletrônica S/A, é formalmente constituído como um instituto de pesquisa tecnológica. Entretanto, pela maneira como opera, ele pode ser considerado como uma nova modalidade de incubadora: enfoca a inovação em produtos, a partir de prospecção tecnológica, ofertando-os para a indústria para que esta os manufature e comercialize.

O restante do artigo está estruturado da seguinte forma: inicialmente, apresenta-se a estrutura analítica do estudo, ou seja, o conceito de incubadoras de empresas, bem como uma tipologia geralmente associada a esta modalidade de infra-estrutura tecnológica. Em seguida, apresenta-se o desenho e o método deste estudo. As evidências empíricas deste trabalho são exploradas, sendo seguidas, finalmente, pelas conclusões do artigo.

\section{ESTRUTURA ANALÍTICA ${ }^{2}$}

Considerando competitividade industrial como um resultado dos contextos nacionais e, mais recentemente, regionais, debate-se a adequabilidade de políticas desenhadas para sustentá-la, tais como a política industrial, de ciência e tecnologia e outras correlacionadas. Tais políticas são, geralmente, focadas em sistemas que, por sua vez, envolvem várias instituições e mecanismos que dão apoio e moldam os caminhos nos quais a inovação ocorre nos setores produtivos e na sociedade como um todo. Observa-se, a partir dos anos 1980, uma alteração nas políticas objetivando a promoção de ajustes estruturais no intuito de estimular a liberalização, a desregulamentação e a competitividade industriais. Pretende-se o fortalecimento das condições estruturais para que as empresas e as indústrias operem em um ambiente mundial cada vez mais competitivo, o qual transcende, do ponto de vista das empresas, a 
formação dos preços, mas prioriza, dentre outros aspectos, a habilidade de inovar. Melhorias contínuas nos produtos, processos, tecnologia e organização são fatores-chave para uma competitividade sustentada em uma economia globalizada (UNCTAD, 1998).

Atualmente, a gama de atores institucionais envolvida com as atividades de inovação, bem como com o seu apoio e avaliação, é bastante acentuada. Dentre esses atores podem ser destacadas empresas, infraestruturas tecnológicas como incubadoras de empresas e parques de ciência e tecnologia, institutos e centros de pesquisa públicos e privados e universidades, para quem a geração, a transferência e o uso de conhecimento e/ou tecnologia constituem atividades fundamentais ou inputs essenciais para a inovação.

Infra-estrutura tecnológica é aqui definida como um conjunto de arranjos institucionais organizados com o objetivo básico de facilitar a disseminação de tecnologia e outros conhecimentos relacionados, de fontes relevantes para as empresas e outras organizações, para auxiliá-las no desenvolvimento de suas competências tecnológicas e na adoção, produção e comercialização de inovações. Estes arranjos institucionais cobrem uma diversidade de mecanismos e processos que servem de apoio para a provisão de serviços, tais como contratos de pesquisa, assistência técnica, certificação, consultoria, treinamento. Assim, as "fontes relevantes" incluem, dentre outras, universidades e seus diversos departamentos, institutos públicos e privados, bancos de dados e empresas de consultoria. Em geral, estes arranjos institucionais são criados e/ou mantidos através da provisão de recursos (financeiros) públicos que permitem que a oferta de serviços acima mencionada ocorra a um preço abaixo do de mercado (Vedovello e Godinho, 2003).

Infra-estruturas tecnológicas, tais como incubadoras de empresas, têm desempenhado um papel cada vez mais importante no cenário da inovação. Busca-se, com essas infra-estruturas, aglutinar políticas e atores sociais distintos e canalizar esforços e recursos com o objetivo de promover um ambiente econômico mais pró-ativo que favoreça o desenvolvimento sócio-econômico mais sustentado e competitivo.

Entretanto, é importante enfatizar que a simples implementação de infra-estruturas tecnológicas não se constitui, por si só, em um fator de sucesso, seja em termos empresariais, setoriais ou nacional. Por um lado, deve-se ter em conta que os processos de novação internos das empresas não são homogêneos, pois assumem diversas formas e fazem uso de diferentes fontes de conhecimento e informação. As empresas têm características próprias, e a sua capacidade de absorção e utilização de novos artefatos sejam materiais, humanos ou financeiros - depende não somente do estágio de desenvolvimento já acumulado, mas também da natureza da tecnologia nos setores produtivos que lhe são afetos e da capacidade de criar sinergias e aproveitar as oportunidades de desenvolvimento apresentadas.

Por outro lado, os fatores de natureza macro e estreitamente vinculados às políticas de ciência e tecnologia (C\&T), de inovação e industrial também afetam o desempenho dessas infra-estruturas tecnológicas. Observa-se que o relacionamento entre os atores institucionais acima mencionados não é linear, sendo sua interação tão intrincada que especialistas ainda apresentam dificuldades em clarificar, de uma forma mais completa, suas funções e ligações.

As incubadoras, com suas pequenas e médias empresas (PMEs) operando em um mesmo segmento produtivo, ou não, e compartilhando um ambiente sócio-econômico e cultural semelhante, podem se constituir em uma nova abordagem de políticas públicas em prol da inovação e da competitividade industrial. Como Kumar \& Kumar (1997) enfatizam, muitas idéias sobre processos, produtos e mesmo negócios potencialmente bons permanecem como tais, e daquelas que são desenvolvidas, poucas se 
tornam bem sucedidas. Essa performance pode ser melhorada a partir da existência de um pacote de serviços e de recursos financeiros - através de várias políticas e programas - providos para/por incubadoras de empresas. Entretanto, a Organização para Cooperação e Desenvolvimento Econômico adverte que a localização de uma incubadora pode ser decisiva para o sucesso de suas operações e mesmo utilidade (OCDE, 1999). Como muitas vezes tal localização se dá em áreas economicamente deprimidas e carentes de outras infra-estruturas que lhe dão suporte, talvez seja operacionalmente mais interessante promover a melhoria do ambiente local para o empreendedorismo antes da instalação de incubadoras.

De forma geral, a 'racionalidade' para a implementação e fortalecimento de incubadoras, segundo a OCDE (1997), pode ser resumido em: (a) promover o desenvolvimento econômico (por exemplo, expansão da base de negócios e diversificação da economia local); (b) promover a comercialização de tecnologias (por exemplo, comercialização de novas idéias, novos artefatos e novos negócios); (c) promover o desenvolvimento de propriedades imobiliárias (property venture); e (d) fomentar o empreendedorismo. Geração de empregos, promoção da auto-suficiência para diversos grupos da população, transferência de tecnologia de universidades e institutos de pesquisa e corporações e compartilhamento de experiências entre os novos empresários e os já estabelecidos são objetivos "secundários" de extrema relevância.

As incubadoras de empresas, que têm sido implementadas em todos os países desenvolvidos e em desenvolvimento, apresentam-se, atualmente, como um locus adequado para abrigar e apoiar as PMEs - eixo central de sua operação -, principalmente as de base tecnológica. Ao prover as PMEs com instalações físicas adequadas e de qualidade, com serviços de apoio compartilhados e com aconselhamento sobre o funcionamento do mercado, sobre tecnologias e seus aspectos, e sobre viabilidade de apoio financeiro, as incubadoras buscam explorar e potencializar os recursos existentes e fomentar as sinergias entre pares. Elas procuram, ainda, criar um ambiente favorável ao surgimento e fortalecimento de novos empreendimentos, ou seja, objetivam tornar as suas incubadas em empresas graduadas bem sucedidas.

A literatura sobre incubadoras de empresas é bastante ampla, grande parte se concentrando no relato das mais variadas experiências. Uma abordagem mais analítica, como a desenvolvida em (Vedovello, 2000, 2001), salienta as dificuldades em se definir incubadoras de empresas de uma forma ampla e única devido, principalmente, à diversidade e heterogeneidade dos modelos em operação. Tal situação é exemplificada pelo contexto europeu, em que a diversidade de estratégias observadas no estabelecimento de incubadoras reflete as diversidades e/ou peculiaridades nacionais dentro do próprio continente. Essas diferenças podem ser encontradas, por exemplo, nos níveis médios de desenvolvimento tecnológico-empresarial e nas políticas nacionais de apoio às PMEs.

A autora discorre sobre os principais grupos de interesse envolvidos com esses empreendimentos (universidades e institutos de pesquisa, empresários e os chamados acadêmicos-empresários, agentes financeiros e capitalistas de risco, e governo, autoridades e agências de desenvolvimento), seus objetivos e fatores determinantes de sucesso: existência de infra-estrutura adequada; universidades e centros/institutos de pesquisa de excelência; empresas e suas características e envolvimento com atividades de pesquisa e desenvolvimento (P\&D); empreendedorismo; e recursos financeiros tanto em termos de financiamento como de capital de risco.

Pode-se pensar em uma incubadora como 'um arranjo inter-institucional com instalações e infraestrutura apropriadas, estruturado para estimular e facilitar a vinculação empresa-universidade (e outras instituições acadêmicas); o fortalecimento das empresas e o aumento de seu entrosamento; o aumento 
da vinculação do setor produtivo com diversas instituições de apoio (além das instituições de ensino e pesquisa, prefeituras, agências de fomento e financiamentos - governamentais e privadas - instituições de apoio às pequenas empresas - como o Serviço Brasileiro de Apoio às Micro e Pequenas Empresas (SEBRAE e outras)' (Amato Neto, 2000). Incubadoras de empresas devem, então, prover às empresas incubadas serviços e recursos compartilhados, ins talações adequadas e infra-estrutura administrativa competente e operacional criando um ambiente pró-ativo ao nascimento, desenvolvimento e consolidação de novos negócios.

Como é sugerido pela Comissão Européia (European Commission, 2001), há uma brecha entre a concepção ou idéia (de um negócio) e a sua transformação em empresa e inserção no mercado. Durante a fase embrionária, há a necessidade de uma infra-estrutura de apoio adequada aos potenciais novos negócios, a qual pode ser suprida pelas incubadoras de empresas. Muitas dispõem, inclusive, de mecanismos de apoio financeiro às suas incubadas. Entretanto, o financiamento desses novos empreendimentos mostra-se como um fator crítico, principalmente quando se trata de países em desenvolvimento. Zedtwitz (2003) desenvolve uma tipologia de incubadoras de empresas não estática, ou seja, elas podem apresentar algumas características que se sobrepõem umas às outras, conforme indicado no Quadro 1.

Quadro 1. Tipologia de incubadoras de empresas

\begin{tabular}{|c|c|}
\hline $\begin{array}{l}\text { Tipos de } \\
\text { Incubadoras }\end{array}$ & Principais Características \\
\hline $\begin{array}{l}\text { Incubadoras } \\
\text { Comerciais } \\
\text { Independentes }\end{array}$ & $\begin{array}{l}\text { Emergem como resultado de atividades prospectivas desenvolvidas por empresários ou } \\
\text { empresas vinculadas ao capital de risco. Gozam de maior liberdade para desenvolver } \\
\text { seus próprios modelos de negócios.Orientadas para o lucro, estas incubadoras se } \\
\text { baseiam fortemente nas suas competências internas e focam suas atividades em uma } \\
\text { dada tecnologia, indústria ou região (por exemplo, software de reconhecimento de } \\
\text { linguagem; mercado japonês). }\end{array}$ \\
\hline $\begin{array}{l}\text { Incubadoras } \\
\text { Regionais }\end{array}$ & $\begin{array}{l}\text { Geralmente estabelecidas pelos governos locais ou organizações com interesses } \\
\text { econômicos e políticos regionais similares, buscando prover espaço e apoio logístico } \\
\text { para os negócios iniciantes em uma dada comunidade. Objetivam acoplar seus } \\
\text { resultados aos interesses delineados pelas políticas públicas: geração de empregos, } \\
\text { aprimoramento da indústria local, ou aprimoramento da imagem pública de uma dada } \\
\text { região. }\end{array}$ \\
\hline $\begin{array}{l}\text { Incubadoras } \\
\text { vinculadas às } \\
\text { Universidades }\end{array}$ & $\begin{array}{l}\text { Universidades podem ser consideradas berço de novas invenções/inovações e } \\
\text { tecnologia de ponta. Estas incubadoras podem, ou não, estar vinculadas a parques } \\
\text { tecnológicos já implantados - e atuam como laboratórios desenhados para aprimorar e } \\
\text { fortalecer a colaboração entre acadêmicos e industrialistas. }\end{array}$ \\
\hline $\begin{array}{l}\text { Incubadoras Intra - } \\
\text { Empresariais }\end{array}$ & $\begin{array}{l}\text { Vinculadas às atividades de P\&D corporativas, têm como principais objetivos lidar com } \\
\text { a descontinuidade tecnológica, incrementar a comunicação entre as funções técnicas e } \\
\text { corporativas, minimizar a inflexibilidade das estruturas organizacionais e gerenciais, e } \\
\text { aprimorar a habilidade de alinhar a visão de longo prazo da corporação com as suas } \\
\text { necessidades de curto prazo. }\end{array}$ \\
\hline $\begin{array}{l}\text { Incubadoras } \\
\text { Virtuais }\end{array}$ & $\begin{array}{l}\text { Diferentemente das incubadoras tradicionais, as virtuais não oferecem espaço físico ou } \\
\text { apoio logístico. Buscam, porém, construir e fortalecer plataformas e redes de acesso a } \\
\text { empresários, investidores e consultores. Esta modalidade de incubadora tem sido } \\
\text { considerada adequada para estágios de negócios muito iniciais e, preferencialmente, } \\
\text { vinculados às tecnológicas de informação. }\end{array}$ \\
\hline
\end{tabular}

Fonte: Baseado em Zedtwitz (2003) 
Scaramuzzi, em seu artigo "Incubators in Developing Countries: Status and Development Perspectives" (World Bank, 2002), apresenta uma excelente síntese sobre a experiência de implantação de incubadoras de empresas em países em desenvolvimento, incluindo o Brasil. No cenário brasileiro, onde as incubadoras de empresas apresentam-se como uma infra-estrutura tecnológica bastante difundida, esse movimento se inicia em meados dos amos 1980. Segundo a ANPROTEC, em seu último levantamento para o ano de 2003, o Brasil já conta com 207 incubadoras instaladas por todo o território, sendo 96 incubadoras na região sul, 71 na região sudeste, 24 na região nordeste, oito na região centro-oeste e oito na região norte (ANPROTEC, 2004). A ANPROTEC classifica as incubadoras brasileiras em quatro tipos: tecnológicas (que abrigam somente empresas de base tecnológica, estimulando parcerias com universidades e centros de pesquisa), tradicionais (não têm como prioridade o estabelecimento de vínculos com universidades e centros de pesquisa), mistas (não possuem restrições quanto ao setor de atividades das empresas) e outras (tais como as incubadoras culturais, as agroindustriais, as cooperativas). Para o período 1999-2003, segundo a Tabela 1, observase uma queda na proporção de incubadoras tecnológicas e tradicionais, um aumento das incubadoras mistas; e o surgimento de incubadoras culturais, agroindustriais e cooperativas.

Tabela 1. Tipos de incubadoras brasileiras - 1999-2003 (em \%)

\begin{tabular}{|l|c|c|c|c|c|}
\hline Tipos de Incubadoras & $\mathbf{1 9 9 9}$ & $\mathbf{2 0 0 0}$ & $\mathbf{2 0 0 1}$ & $\mathbf{2 0 0 2}$ & $\mathbf{2 0 0 3}$ \\
\hline Tecnológicas & 64 & 59 & 55 & 57 & 52 \\
\hline Tradicionais & 22 & 23 & 31 & 29 & 25 \\
\hline Mistas & 14 & 18 & 14 & 14 & 20 \\
\hline $\begin{array}{l}\text { Outras (culturais, agroindustriais e } \\
\text { de cooperativas) }\end{array}$ & 0 & 0 & 0 & 0 & 3 \\
\hline
\end{tabular}

Fonte: ANPROTEC (2004).

Embora as tipologias acima sejam ricas em termos de possibilidades de descrições de tipos de incubadoras de empresas, elas não são suficientemente abrangentes para captar um tipo de incubadora caracterizada por se originar como uma incubadora que nasce dentro de uma corporação e tornar-se, rapidamente, uma incubadora independente com foco exclusivo no desenvolvimento de projetos tecnológicos - ou seja, uma incubadora de idéias, de inovação tecnológica - operando em um contexto de economia emergente e/ou área em desenvolvimento. Por essa razão, faz-se necessário ampliar as tipologias apresentadas a fim de incorporar experiências desta natureza.

\section{MÉTODO}


Para examinar essa modalidade de incubadora, com adequado nível de detalhe e profundidade, foi necessária a coleta de evidências empíricas primárias, principalmente qualitativas, sobre as atividades tecnológicas e os vários processos e mecanismos de aprendizagem usados na organização. Essas evidências foram obtidas a partir de fontes múltiplas - técnicos, engenheiros, gerentes e diretores do Genius Instituto de Tecnologia, em sua sede em Manaus (AM) e também em Campinas (SP), através de trabalho de campo realizado entre outubro/2002 e dezembro/2003. Tais fontes foram acessadas com base em técnicas variadas: entrevistas formais, encontros casuais, consulta à documentação (procedimentos, arquivo técnico, dados históricos e etc) e observação direta.

Considerando que uma coleta de dados para um estudo dessa natureza não pode ser feita com base em apenas uma visita à organização estudada, independentemente do número de entrevistados (Dutrènit, 2000; Figueiredo, 2001, 2004), a coleta de evidências empíricas para substanciar este estudo envolveu um "processo" que se desenvolveu ao longo de seis etapas de interação com a organização, em ocasiões diferentes (outubro/2002 a dezembro/2003), a saber:

(1) Entrevista realizada por um dos autores, na Gradiente Eletrônica S/A, com um dos dirigentes fundadores do Genius;

(2) Entrevistas coletivas e individuais, realizadas por um dos autores, com líderes de projetos e pesquisadores no Genius, seguidas de visitas às instalações e aos laboratórios;

(3) Entrevista realizada pelos dois autores com um dos dirigentes fundadores do Genius;

(4) Entrevistas coletivas e individuais, realizadas pelos dois autores, com líderes de projetos e pesquisadores no Genius, seguidas de visitas às instalações e aos laboratórios. Esta etapa também envolveu uma apresentação do Genius aos autores, sobre a concepção e atividades do Instituto. Isto possibilitou obter informações mais detalhadas sobre suas operações. Além disso, houve a apresentação de um seminário, por um dos autores, para os colaboradores do Genius, o que proporcionou uma interação ainda mais intensa com a organização pesquisada.

(5) Redação do relatório preliminar do estudo baseado na sistematização dos dados coletados. Este relatório, enviado para dois dirigentes do Genius para validação, retornou com informações adicionais;

(6) Entrevista realizada por um dos autores, com um líder de projeto, para confirmação de análise e validação final dos resultados.

Utilizamos aqui o método de estudo de caso individual (Yin, 1994; Leonard-Barton, 1995), que permite o exame de uma incubadora de inovação com adequado nível de profundidade e de detalhe. Logo, estamos interessados em contribuir para expandir a compreensão desta nova modalidade de incubadora. Ou seja, a nossa preocupação aqui é com a generalização analítica.

\section{A EXPERIÊNCIA DO GENIUS INSTITUTO DE TECNOLOGIA: PONTOS FORTES E FRACOS}


A Gradiente Eletrônica S/A, criada em 1964, é uma empresa brasileira, voltada para o mercado nacional, e dedicada à produção de bens eletro-eletrônicos de consumo (som, DVD, televisores). Após um processo de reestruturação, a empresa busca, além das áreas tradicionais de atuação, tais como a produção de equipamentos eletrônicos e de entretenimento (videogames e jogos eletrônicos), ampliar o seu escopo de atuação abrangendo a produção de computadores (projetado para operar interativamente como um aparelho de DVD e receber sinais das emissoras abertas e a cabo como se fosse um televisor comum) e a prestação de serviços, como no caso do Gradiente in Motion (projetado para servir como uma espécie de secretária virtual que poderá fornecer informações sobre trânsito, tempo, esportes e loterias). Estes setores, mundialmente, têm apresentado algumas características interessantes, cabendo destacar: (1) setor dominado por grandes corporações multinacionais com grande capacidade de inovação de produto e processo; (2) setor dominado pela rápida mudança tecnológica. Dentro desse contexto - e, certamente, face às pressões competitivas nessa indústria para a implementação de atividades inovadoras em produto, processo, e organização da produção - a Gradiente criou, em novembro de 1999, o Genius Instituto de Tecnologia ${ }^{3}$.

O Genius Instituto de Tecnologia foi concebido, originalmente, como um instituto de pesquisa privado, sem fins lucrativos e com gestão independente. Seu objetivo central era apoiar as atividades de pesquisa e desenvolvimento (P\&D) da Gradiente, contando, para isso, com aportes financeiros para serem investidos ao longo dos cinco primeiros anos ${ }^{4}$. Nessa fase inicial, o Genius pode, então, ser considerado como uma incubadora pertencente a uma corporação, exclusivamente focada no desenvolvimento tecnob́gico.

Entretanto, esta fase foi rapidamente superada e o Genius tornourse uma instituição, de fato, independente da Gradiente, ou seja, uma incubadora independente, mantendo sua característica exclusiva de foco no desenvolvimento tecnológico. Como enfatizado em diferentes entrevistas, o Genius transformourse em uma "incubadora tecnológica para inovação", que prioriza a maturação de uma tecnologia e não a viabilização de uma nova empresa operando com tecnologias já existentes.

O Genius foca a sua ação em dois vetores-chave: (a) o desenvolvimento de alguns produtos/processos de alta tecnologia criteriosamente selecionados e que possam ser acoplados à política de propriedade industrial (gerando maior independência face ao mercado internacional); e (b) ao atendimento eventual de demandas tecnológicas do próprio mercado. Em outras palavras, o modelo proposto pelo Genius, ao estimular esse tipo de comportamento, pressupõe maior pró-atividade e independência do mercado tecnológico já constituído. Esse aspecto é de fundamental relevância e distingue esta modalidade de incubadora de outras tradicionais.

Os modelos de incubadora tecnológica tradicionais carecem, por várias razões, de apoio financeiro e logístico mais adequados afetando, consequientemente, o apoio que possa ser dado às empresas incubadas. As empresas incubadas, por sua vez, também devido às suas limitações financeiras, acabam sendo 'obrigadas' a se voltarem para o mercado em busca de receitas que garantam a sua sobrevivência. Em outras palavras, estas pequenas empresas se tornam cativas de um nicho específico do mercado (muitas vezes representado por um único cliente), cuja demanda é, geralmente, de curto prazo. Esse comportamento pode, eventualmente, tornar tais empresas incapazes de reverter essa tendência. Ao longo do tempo, elas podem distanciar-se, cada vez mais, de seu foco de negócios inicial. Ou seja, a empresa se torna incapaz de criar um portfólio de produtos/processos ou serviços com o qual ela possa atuar de maneira pró-ativa em relação ao mercado.

A partir de janeiro de 2002, o Genius definiu como política de atuação concentrar-se em um número limitado de tecnologias que, após criterioso processo de seleção (road map tecnológico) e estudos de 
viabilidade técnica e econômica (EVTEs), são trabalhadas para futura comercialização. Por exemplo, durante o ano de 2001, após realizar mais de 30 análises conjunturais, EVTEs e protótipos físicos, a equipe de tecnologia do Genius elegeu o reconhecimento de voz em português e espanhol para acionar aparelhos usados cotidianamente como automóveis, computadores ou eletrodomésticos (ASR automatic speech recognition) como a primeira tecnologia-alvo para desenvolvimento.

Em paralelo, o Genius passou a participar do desenvolvimento de tecnologia para os telefones celulares da Siemens mundial. Vale dizer que o Genius teve que desenvolver um trabalho de persuasão junto à Siemens Corporation para que essa inovação fosse desenvolvida fora do seu departamento de P\&D corporativo, localizado na Alemanha. Conforme menciondo em várias entrevistas no Genius, essa conquista é muito significativa, pois trata-se de um fornecimento de uma inovação a uma corporação transnacional altamente inovadora à partir de uma jovem organização localizada em um país e em uma área em desenvolvimento.

\section{(a) Pontos Fortes}

O presente estudo encontrou evidências sobre duas características-chave do Genius que estão associadas ao seu funcionamento: recursos humanos altamente qualificados e parcerias.

1. Recursos humanos altamente qualificados. A fim de desenvolver a sua base de conhecimento inicial, o Instituto atraiu profissionais de várias áreas (por exemplo, engenheiros, cientistas de computação, lingüistas) de pelo menos três fontes principais. A primeira, proveniente da própria Gradiente, mais especificamente de sua unidade de desenvolvimento/engenharia de produto, tanto da sua unidade em Manaus como de São Paulo. A segunda, referente à contratação dos melhores engenheiros e técnicos da área de design e desenvolvimento de produtos da Sharp do Brasil, através da sua unidade em Manaus ${ }^{5}$. A terceira fonte baseourse no recrutamento de pesquisadores - mestres, doutorandos e doutores brasileiros 'repatriados' de outros países como Holanda, Bélgica e Canadá, bem como recrutados de 14 estados brasileiros. Por exemplo, vale destacar o caso da contratação de um brasileiro, doutorado pela Universidade de Tóquio e líder de projeto na empresa belga Lerneut \& Hauspie (L\&H) que vinha atuando na área de desenvolvimento da tecnologia de reconhecimento de voz e que faliu em 2001. Além disso, foram recrutados pesquisadores estrangeiros de quatro países. Atualmente, o Genius conta com aproximadamente 100 colaboradores - dos quais mais de $70 \%$ são $\mathrm{PhDs}$, doutorandos, mestres e mestrandos - atuando tanto na área de pesquisa de produto como nas áreas de testes em seus laboratórios. Essa estratégia de aquisição de uma variedade de profissionais ilustra a importação de uma variada base de conhecimento tácito que certamente contribui para acelerar o desenvolvimento de atividades inovadoras.

2. Parcerias. Além de contar com o seu quadro próprio, o Genius tem estabelecido parcerias a fim de complementar a sua base de conhecimento para desenvolver as suas atividades inovadoras. O Quadro 2 descreve os parceiros e as atividades desenvolvidas em conjunto com o Genius. 
INCUBADORA DE INOVAÇÃO: QUE NOVA ESPÉCIE É ESSA?

Conceição Vedovello - Paulo N. Figueiredo

\section{Quadro 2. Principais parcerias estabelecidas pelo Genius Instituto de Tecnologia}

\begin{tabular}{|c|c|c|}
\hline Instituição Parceira & $\begin{array}{c}\text { Área específica da instituição } \\
\text { parceira }\end{array}$ & $\begin{array}{c}\text { Atividade desenvolvida com o } \\
\text { Genius }\end{array}$ \\
\hline Ministério da Ciência e Tecnologia & $\begin{array}{l}\text { Secretaria de Política de } \\
\text { Informática }\end{array}$ & $\begin{array}{l}\text { Pesquisa e desenvolvimento em } \\
\text { informática e eletrônica de } \\
\text { consumo; elaboração do Programa } \\
\text { Nacional de Microeleterônica. }\end{array}$ \\
\hline $\begin{array}{l}\text { Superintendência da Zona Franca } \\
\text { de Manaus (SUFRAMA) }\end{array}$ & $\begin{array}{l}\text { Departamento de Promoção de } \\
\text { Investimentos }\end{array}$ & $\begin{array}{l}\text { Pesquisa e Desenvolvimento em } \\
\text { Informática e Eletrônica de } \\
\text { consumo; organização do } \\
\text { workshop "The Global Integrated } \\
\text { Circuit Design Market". }\end{array}$ \\
\hline $\begin{array}{l}\text { Financiadora de Estudos e Projetos } \\
\text { (FINEP) }\end{array}$ & & $\begin{array}{l}\text { Pesquisa e Desenvolvimento nas } \\
\text { áreas de reconhecimento } \\
\text { automático de voz e portais de voz. }\end{array}$ \\
\hline Fundação CPqD & & $\begin{array}{l}\text { Pesquisa e desenvolvimento na } \\
\text { área de televisão digital terrestre. }\end{array}$ \\
\hline $\begin{array}{l}\text { Centro Federal de Educação } \\
\text { Tecnológica do Maranhão } \\
\text { (CEFET/MA) }\end{array}$ & & $\begin{array}{l}\text { Estudo de amplificadores para } \\
\text { aplicações em áudio. }\end{array}$ \\
\hline Universidade do Amazonas & $\begin{array}{l}\text { Departamento da Ciência da } \\
\text { Computação }\end{array}$ & $\begin{array}{l}\text { Implementação conjunta do } \\
\text { primeiro mestrado em Informática } \\
\text { da Região Norte. }\end{array}$ \\
\hline $\begin{array}{l}\text { Universidade Estadual de } \\
\text { Campinas }\end{array}$ & $\begin{array}{l}\text { Núcleo Interdisciplinar de } \\
\text { Comunicação Sonora } \\
\text { Departamento de Engenharia de } \\
\text { Computação e Automação }\end{array}$ & 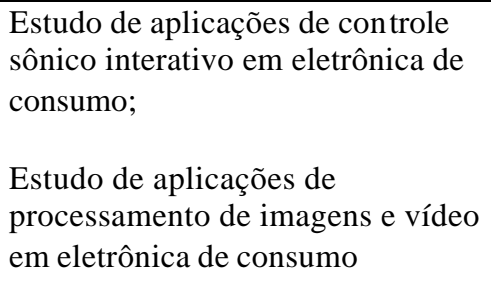 \\
\hline Universidade Federal da Paraíba & $\begin{array}{l}\text { Departamento de Sistemas e } \\
\text { Computação }\end{array}$ & $\begin{array}{l}\text { Projeto de circuitos integrados para } \\
\text { a transmissão sem fio de áudio de } \\
\text { alta qualidade. }\end{array}$ \\
\hline $\begin{array}{l}\text { Universidade Federal do Rio de } \\
\text { Janeiro }\end{array}$ & $\begin{array}{l}\text { Laboratório de Processamento de } \\
\text { Sinais }\end{array}$ & $\begin{array}{l}\text { Consultoria na área de } \\
\text { processamento digital de sinais }\end{array}$ \\
\hline Universidade de São Paulo & Instituto de Física de São Carlos & $\begin{array}{l}\text { Proposta conjunta do Instituto } \\
\text { Multidisciplinar de Materiais } \\
\text { Poliméricos ao Programa Institutos } \\
\text { do Milênio do CNPq. }\end{array}$ \\
\hline
\end{tabular}

Fonte: Genius Instituto de Tecnologia.

Para identificar potenciais parceiros que possam complementar a base de conhecimento em atividades inovadoras diversas, o Genius desenvolveu a figura do "promotor Genius" - responsável por facilitar 
os contatos da organização com instituições aptas a estabelecer parcerias. Atualmente, o Genius conta com uma rede de 26 "promotores" e instituições no Brasil e 12 internacionais.

Dentre as parcerias já estabelecidas, cabe destacar a atuação conjunta com a empresa americana Speechworks, parceria esta que buscou fortalecer o núcleo para o desenvolvimento da plataforma de reconhecimento de voz em português e espanhol. Adicionalmente, o Genius desenvolveu uma parceria com a Motorola para trabalhar em um sistema de transmissão de áudio via rádio, que dispensa os fios e cabos.

As evidências sugerem que essas parcerias estabelecidas com instituições e empresas nacionais e transnacionais parecem decorrer de uma característica da empresa-mãe - Gradiente - que, ao longo de sua existência, acumulou experiência de consolidar parcerias estratégicas, tais como JVC, Nintendo e Nokia. Além disso, a Gradiente parece ter imprimido no Genius o seu estilo organizacional empreendedor, valendo-se de lideranças em vários níveis corporativos, cujo comportamento tem impulsionado a organização para o seu engajamento em atividades inovadoras (veja, por exemplo, Silva, 2002).

\section{(b) Pontos Fracos}

Como já mencionado anteriormente, o Genius Instituto de Tecnologia constitui-se em um empreendimento jovem e inovador apresentando, portanto, pontos fracos típicos oriundos desse tipo de projeto. Adicionalmente, enfrenta os desafios naturais de operar em um contexto de economia emergente, mais especificamente, em uma área de industrialização recente no Brasil (Pólo Industrial de Manaus). As evidências coletadas neste estudo sugerem quatro principais pontos fracos que se apresentam ao Genius para o desenvolvimento de suas atividades:

1. Substituição do Conselho Deliberativo do Genius Instituto de Tecnologia. Embora sendo financeiramente independente da Gradiente Eletrônica S/A (empresa que lhe deu origem), o Conselho Deliberativo do Genius era composto, até recentemente, por cinco executivos da empresa, fato que comprometia a organização perante o mercado, provocando uma percepção equivocada. Em outubro de 2003, o Conselho Deliberativo passou por uma profunda modificação sendo, desde então, composto por um representante dos institutos de pesquisa, dois representantes de universidades, um representante do mercado e um representante da empresa Gradiente Eletrônica S/A. Espera-se que essa nova composição possa dar maior independência ao Genius, ampliando as suas possibilidades junto ao mercado.

2. Ampliação do portfólio de clientes. Dada a sua origem - a empresa Gradiente Eletrônica S/A - o Genius pode ser alvo de desconfiança por parte de outros potenciais clientes. As alterações implantadas no Conselho Deliberativo (como explicado acima) devem dirimir parte substancial desse ponto fraco. A parceria com a Siemens - primeiro cliente efetivo do Genius - constitui se em um 'piloto' para avaliar a resistência do mercado a sua origem corporativa. Essa parceria foi estabelecida não sem grande esforço de convencimento por parte do Genius em relação às suas credenciais. As atividades se iniciaram através de um projeto de pesquisa modesto em termos tecnológicos, por exigência da própria Siemens. Durante os trabalhos, o Genius foi auditado por uma equipe de consultoria da própria 
Siemens, da Alemanha, auditoria essa baseada em quatro estágios e que objetivava não somente verificar a idoneidade física e lógica do Instituto, mas também os custos e o atendimento ao cronograma de atividades. Os resultados obtidos consideraram o Genius como de baixo risco, ou seja, tecnicamente competente e estrategicamente confiável.

3. Ausência de referências ao próprio Genius. A concepção do Genius Instituto de Tecnologia pode ser considerada como pioneira no contexto brasileiro, com poucos institutos dessa natureza. Ao contrário da experiência americana que conta com mais de quinze mil empreendimentos desta natureza, o Genius não tem referências fortes - em termos locais e nacionais - de institutos similares voltados para a inovação tecnológica, com a mesma filosofia (foco na geração de tecnológicas próprias) em que se espelhar e com quem estabelecer sinergias e trocar experiências.

4. Necessidade de auto-sustentação. Embora contando com recursos financeiros que o suporte por alguns anos e lhe provê de certa independência, as evidências sugerem que o Genius luta pela sua autosustentação. Nesse sentido, corre o risco de sacrificar o futuro pelo presente e há que se evitar que os problemas cotidianos absorvam as atenções e esforços da sua equipe. $O$ grande desafio que se apresenta é conjugar a necessidade de prospectar tecnologias com a necessidade cotidiana de entregar projetos inovadores, buscar clientes, estabelecer parcerias e aprofundar a sua competência técnica e organizacional.

\section{DISCUSSÃO E CONCLUSÕES}

Ao examinar a experiência do Genius como uma incubadora de inovação, este artigo dá um passo à frente em relação à literatura existente, cujas tipologias convencionais não captam experiências desta natureza. Isso é importante porque abre a possibilidade de ampliar a utilização do mecanismo (incubadora) para o desenho de estratégias industriais governamentais orientadas para o fortalecimento das infra-estruturas de tecnologia e inovação (sistemas locale nacional de inovação), principalmente no contexto de economias emergentes e/ou áreas em desenvolvimento, como é o caso de Manaus.

As incubadoras de empresas têm desempenhado um papel cada vez mais importante no cenário da inovação, aglutinando políticas e atores sociais distintos e canalizando esforços e recursos com o objetivo de promover um ambiente econômico mais pró-ativo que favoreça o desenvolvimento sócioeconômico mais sustentado e competitivo. Os seus modelos mais convencionais têm buscado prover suas empresas incubadas com instalações físicas adequadas e de qualidade, com serviços de apoio compartilhados e com aconselhamento sobre o funcionamento do mercado, sobre tecnologias e seus aspectos, e sobre a viabilidade de apoios financeiros.

Entretanto, esses modelos carecem de apoio financeiro e logístico mais adequados afetando, conseqüentemente, o apoio que possa ser dado às empresas incubadas. Estas, por sua vez, também devido às suas limitações financeiras, acabam sendo 'induzidas' a se voltar para o mercado em busca 
de receitas que garantam a sua sobrevivência. Ou seja, ao se tornarem cativas de um nicho específico do mercado, atendendo demandas de curto prazo, estas pequenas empresas acabam por comprometer o próprio foco inicial de seus negócios.

A experiência do Genius, que conta com logística e aporte de recursos financeiros 'estáveis' por um certo período de tempo, prioriza o foco central de sua atuação - o desenvolvimento de tecnologias e inovação - sustentado por dois pilares essenciais para esse tipo de empreendimento: (i) a atração e manutenção de profissionais altamente qualificados e (ii) o estabelecimento de parcerias para complementar a sua base de conhecimento, fortalecendo, desta forma, suas capacidades para desenvolver atividades ino vadoras.

O Genius se constitui em um empreendimento muito jovem e inovador, enfrentando, portanto, desafios típicos de um empreendimento pioneiro em um contexto de economia emergente. A sua sustentabilidade, no longo prazo, dependerá da superação dos seus pontos fracos, ou seja, da sua capacidade de manter e aprofundar a base de conhecimento que lhe dá sustentação, de criar e manter sinergias com seus pares e outras organizações, de gerar novos produtos, de atrair e ampliar sua carteira de clientes e de gerar recursos próprios de maneira competitiva. Em outras palavras, o Genius necessita fortalecer, de maneira continuada, a sua estrutura interna com independência para ampliar suas oportunidades de mercado.

De uma forma geral, a experiência do Genius contrib ui para a expansão do entendimento sobre as bases de sustentação e o funcionamento de incubadoras de inovação. Auxilia, ainda, na geração de insumos para a calibração de estratégias empresariais e governamentais relativas à criação e/ou aceleração do desenvolvimento tecnológico em setores industriais específicos. Mais especificamente, as evidências e análise que emergem deste trabalho contribuem para um maior entendimento da base organizacional e gerencial de uma incubadora de inovação.

\section{NOTAS}

${ }^{1}$ Este estudo é parte de um projeto de pesquisa mais abrangente, desenvolvido pelos autores de setembro de 2002 a dezembro de 2003, que examinou a interação entre a infra -estrutura tecnológica e setores industriais específicos no Brasil. Esse projeto foi implementado no âmbito do Programa de Pesquisa em Aprendizagem Tecnológica e Inovação Industrial no Brasil, da Escola Brasileira de Administração Pública e de Empresas (EBAPE) da Fundação Getulio Vargas (FGV). Os autores são especialmente gratos ao professor Bianor Scelza Cavalcanti, diretor da EBAPE, e à professora Deborah Moraes Zouain, chefe do Centro de Formação Acadêmica e Pesquisa da EBAPE, pelo constante apoio e encorajamento às atividades desse Programa de Pesquisa. Somos extremamente gratos ao Instituto Supenior de Administração e Economia (ISAE/FGV), em Manaus, particularmente ao seu superintendente Lincoln Campos, pelo apoio durante as nossas atividades de trabalho de campo em Manaus. Queremos expressar nossa profunda gratidão aos dirigentes e pesquisadores do Genius Instituto de Tecnologia pela sua cooperação, atenção e paciência durante nossas insistentes visitas e entrevistas.

${ }^{2}$ Desenvolvida a partir de Vedovello (2001).

${ }^{3}$ É importante salientar que para a constituição do Genius Instituto de Tecnologia, a Gradiente Eletrônica S/A usou recursos financeiros advindos da venda de sua participação na fábrica de telefones celulares que mantinha em Manaus, em sociedade com a Nokia Corporation. Esses recursos financeiros foram utilizados para liquidar parte das dívidas da Gradiente, bem como realizar investimentos para a criação do Instituto. 
${ }^{4}$ Além dos recursos privados, o Instituto também se beneficia de recursos públicos advindos, por exemplo, dos benefícios fiscais da Lei da Informática e dos Fundos Setoriais. Vale dizer que o acesso aos Fundos Setoriais deve-se ao fato do Genius ser o primeiro Instituto privado brasileiro de pesquisa e desenvolvimento (P\&D) a receber credenciamento permanente do Comitê da Área de Tecnologia da Informação (CATI).

5 Segundo Ariffin e Figueiredo (2003), até meados dos anos 1990, a Sharp do Brasil era a única empresa brasileira do setor eletro-eletrônico a desenvolver competências tecnológicas próprias para realizar atividades de design e desenvolvimento de produto por meio de sua unidade em Manaus. Porém, ao final dos anos 1990, a Sharp passou por uma profunda crise financeira que forçou a empresa a encerrar as suas atividades manufatureiras e, conseqüientemente, de desenvolvimento de produto.

\section{REFERÊNCIAS BIBLIOGRÁFICAS}

AMATO NETO, J. Redes de cooperação produtiva e clusters regionais - oportunidades para as pequenas e médias empresas. Editora Atlas S. A./Fundação Vanzolini, 2000.

ANPROTEC (Associação Nacional das Entidades Promotoras de Empreendimentos de Tecnologia Avançada) - Panorama 2003 - Disponível em <www.anprotec.org.br>. Acesso em maio 2004

ARIFFIN, N.; FIGUEIREDO, P. N. Internacionalização de competências tecnológicas. Implicações para estratégias governamentais e empresariais para a competitividade e a inovação da indústria eletrônica no Brasil. Rio de Janeiro: Editora da Fundação Getulio Vargas, 2003.

AYDALOT, P.; KEEBLE, D. (eds.). High technology industry and innovative environments: the European experiences. Routledge, 1988.

COHEN, J.; SIMMIE, J. Innovation and technopolis planning in Britain and France. National Institute of Economic and Social Research, Single European Market Initiative, 1991 (Working Paper, 4).

CURRIE, J. Science parks in Britain - their role for the late 1980s. CSP Economic Publications, 1985.

DORFMAN, N. S. Route 128: the development of a regional high technology economy. Research Policy, v. 12, n. 6, p. 299-316, 1983.

DUTRÉNIT, G. Learning and Knowledge Management in the Firm. From Knowledge Accumulation to Strategic Capabilities. Edward Elgar: Cheltenham, UK \& Northampton, MA, 2000.

EUROPEAN COMMISSION. Innovation and technology transfer. Mar. 2001.

EUROPEAN INNOVATION MONITORING SYSTEM (EIMS), Comparative study of Science Parks in Europe: Keys to a Community Innovation Policy. European Commission, Directorate General XIII, The Innovation Programme, 1996 (EIMS Publication, 29).

FIGUEIREDO, P. N. Technological Learning and CompetitivePerformance. Cheltenham, UK, and Northampton, MA, USA: Edward Elgar Publishing, 2001. 
FIGUEIREDO, P. N. Pesquis a empírica sobre aprendizagem tecnológica e inovação industrial: alguns aspectos práticos de desenho e implementação. In: Vieira, M. M e Zouain, D. (Orgs.), Pesquisa qualitativa em administração. Rio de Janeiro: Editora FGV, 2004.

KUMAR, U.; KUMAR, V. Incubating technology: best practices. Canadá: Federal Partners in Technology Transfer, National Research Council, 1997.

LALKAKA, R. Lessons from international experience for the promotion of business incubation systems in emerging economies. Paper commissioned by the Small and Medium Industries Branch. Unido: Small and Medium Enterprises Programme, 1997.

LEONARD-BARTON, D. A. Methodology for Case Studies. Synergistic use of a longitudinal single site with replicated multiple sites. In: Huber, G. P.\& A. van de Ven (Orgs.), Longitudinal Field Research Methods. Studying Processes of Organizational Change. Sage Publications: London, 1995.

LUNDVALL, B.- $\AA$. et al. National systems of production, innovation and competence building, Research Policy, n. 2, p. 213-31, 2002.

MASSEY, D.; QUINTAS, P.; WIELD, D. High tech fantasies: science parks in society, science and space. Routledge, 1992.

OECD. Technology Incubators: nurturing small firms. Paris, 1997.

OECD. Business incubation: international case studies. Paris, 1999.

SCARAMUZZI, E. Incubators in Developing Countries: status and development perspectives. InfoDev Program, Washington D.C.: The World Bank, 2002, mimeo.

SILVA, L. S. Acumulação de Competências Tecnológicas e suas Implicações para o Aprimoramento em Indicadores de Performance Operacional na Gradiente Eletrônica S.A. Dissertação (Mestrado) - E Escola Brasileira de Administração Pública e de Empresas, Fundação Getulio Vargas, Rio de Janeiro.

UNCTAD. Promoting and sustaining SMEs clusters and networks for development. Trade and Development Board TD/COM.3/EM.5/2, 1998.

VEDOVELLO, C.; GODINHO, M., Business Incubators as a Technological Infrastructure for Supporting Small Innovative Firms Activities, International Journal of Entrepreneurship and Innovation Management, v. 3, n. 1/2, p. 4-21, 2003.

VEDOVELLO, C. Aspectos Relevantes de Parques Tecnológicos e Incubadores de Empresas. Revista do BNDES, v. 7, n. 14, p. 273-300, 2000.

VEDOVELLO, C. Criação de infra-estruturas tecnológicas: a experiência brasileira de incubadoras de empresas. Revista do BNDES, v. 8, n. 16, p. 183-214, 2001.

WORLD BANK. World development report on knowledge for development. Oxford University Press, 1999.

YIN, R.K. Case study research - design and methods. Applied Social Research Methods Series, v. 5, $2^{\mathrm{a}}$ edição. USA: Sage Publications, 1994. 
ZEDTWITZ, M. Classification and management of incubators: aligning strategic objectives and competitive scope for new business facilitation. International Journal of Entrepreneurship and Innovation Management, v. 3, n. 1/2, 2003.

\section{Artigo recebido em 15.03.2004. Aprovado em 16.07.2004.}

\section{Dados Autores:}

\section{Conceição Vedovello}

Ph.D. em Estudos de Política de Ciência e Tecnologia pelo SPRU - Science and Technology Policy Research, University of Sussex, Reino Unido

Interesses de pesquisa nas áreas de política de ciência e tecnologia e sua avaliação, infra-estrutura tecnológica e sua avaliação e mecanismos de transferência de tecnologia e sua avaliação.

E-mail: connie@ finep.gov.br

Endereço: Praia do Flamengo, 200, 13ªndar, Flamengo, Rio de Janeiro - RJ, 22210-030.

\section{Paulo N. Figueiredo}

Ph.D. em Gestão da Tecnologia e da Inovação pelo SPRU - Science and Technology Policy Research, University of Sussex, Reino Unido

Interesses de pesquisa nas áreas de aprendizagem tecnológica, desenvolvimento de competências técnicas e organizacionais e performance competitiva em empresas industriais de economias emergentes.

E-mail: pnf@fgv.br

Endereço: Praia de Botafogo, 190, sala 510, Botafogo, Rio de Janeiro - RJ, 22250-900. 